\title{
p16 Expression as a Surrogate Marker for HPV Infection in Esophageal Squamous Cell Carcinoma can Predict Response to Neo-Adjuvant Chemotherapy
}

\author{
Rajeev Kumar ${ }^{1,2}$, Sankar Kumar Ghosh ${ }^{2}$, Akalesh Kumar Verma1, Anuradha \\ Talukdar ${ }^{1}$, Monoj Kumar Deka ${ }^{3}$, Mira Wagh ${ }^{1}$, H.M. Iqbal Bahar ${ }^{1}$, Ritesh \\ Tapkire $^{1}$, Kali Pankaj Chakraborty ${ }^{1}$, R. Ravi Kannan ${ }^{1 *}$
}

\begin{abstract}
Background: Esophageal squamous cell carcinoma (ESCC) is a common cancer in the north east of India. The present study concerned the prevalence of human papilloma virus (HPV) in the ESCC in north eastern India and its impact on response to chemotherapy. Materials and Methods: p16 expression, a surrogate marker for HPV infection was assessed in 101 pre-treatment biopsies of locally advanced ESCC, reported from a comprehensive cancer centre in north east India, using immunohistochemistry. All patients received neo-adjuvant chemotherapy. Response was assessed clinically and histopathologically with attention to p16 expression. Results: p16 was expressed in $22 \%$ of ESCC (22 out of 101) and was more prevalent in patients who were more than 45 years of age $(\mathrm{P}=\mathbf{0 . 0 4 8})$. p16 positive tumors appeared more commonly in the upper $2 / 3$ of the thoracic esophagus (18 in 22). Nine of the $22(41 \%)$ p16 positive tumors achieved pathologic complete response following neo-adjuvant chemotherapy $(P=0.008)$. There was a trend towards reduced mortality in this group $(P=0.048)$. Some 9 of the $20(45 \%)$ patients who achieved pathologic complete response were p16 positive. Conclusions: Expression of p16 in ESCC correlates with higher rate of pathologic complete remission in patients undergoing neo adjuvant chemotherapy and could be a predictive marker for response assessment.
\end{abstract}

Keywords: p16 - HPV - esophageal squamous cell carcinoma - response assessment - neo adjuvant chemotherapy

Asian Pac J Cancer Prev, 16 (16), 7161-7165

\section{Introduction}

Several reported epidemiological studies worldwide have identified the high risk countries for esophageal cancer that included the People's Republic of China, Singapore, former USSR, Iran, Puerto Rico, Chile, Brazil, Switzerland, France, and South Africa (Syrjanen, 2002). It is the eighth most common cancer worldwide, with an estimated 456,000 new cases in 2012, and the sixth most common cause of cancer related death with an estimated 400,000 deaths (IARC, 2014). India is among those Asian countries that shares the high burden of esophageal cancer (Chung et al., 2010). North eastern India falls into region adjoining southern edge of the Asian esophageal cancer belt.

The age adjusted incidence rates (AARs) of 7.6 and 5.1 per 1,00,000 of esophageal cancer were observed in India as compared to USA (4.9 and 1.4) for males and females, respectively (Ali et al., 2011). In north eastern India, where the tobacco and areca nut use is rampant, incidence of ESCC is relatively high. The age adjusted incidence rates of esophageal cancer per 100,000 persons for males and females from the high-risk region of Northeast India have been 71.4 in males and 30.2 in females from East Khasi Hills of Meghalaya state as reported by National Cancer Registry Program (NCRP), Indian Council of Medical Research (ICMR, NCRP 2013). Population based cancer registries in other parts of north east India reported higher incidence of esophageal cancer. The AARs observed in Meghalaya, Kamrup Urban district and Cachar district are 46.2, 27 and 11.7 in males whereas 19.8, 18.3 and 8.3 in females respectively (NCRP 2013).

Esophageal cancer comprises of two main histopathology types, adenocarcinoma and squamous cell carcinoma. Both types have their specific characteristics in terms of etiology, epidemiology, molecular, therapeutic and prognostic aspects. Esophageal adenocarcinoma (EA) is associated with gastro-esophageal reflux and obesity, whereas esophageal squamous cell carcinoma (ESCC) is associated with use of tobacco and alcohol (Lagergren and Lagergren 2010). ESCC is more common with an approximate percentage of $80 \%$ than EA (20\%) in India (Pramesh et al., 2015).

Syrjanen in 1982 had reported human papillomavirus 
(HPV) infection as one of the possible etiological factors in ESCC. However HPV positivity using immunohistochemistry in ESCC is conflicting that ranges between 10 to $40 \%$ of HPV in tumor tissues (Syrjanen, 2002; Michaelsen et al., 2014).

p16 expression is usually used as a surrogate marker for detecting HPV infection due to its high sensitivity, low cost and simplicity (Kalof and Cooper, 2006; Naggar and Westra, 2012).

In India, the incidence of esophageal carcinoma differs from region to region and the association of HPV infection in ESCC has been demonstrated in some of studies (Shukla et al., 2009; Mohiuddin et al., 2013; Vaiphei et al., 2013).

HPV infection has been reported to correlate with the clinical outcome in different squamous cell malignancies. p16 expression that is correlated with HPV infection was shown as marker for responder and better prognosis among head and neck squamous cell carcinoma patients who underwent radiotherapy (Lassen et al., 2009). Chemotherapy could be omitted in this group of patients. Similarly there is some evidence to suggest that high p16 expression correlates with favourable prognosis in esophageal squamous cell carcinoma as well (Sturm et al., 2001; Cao et al., 2014).

There exist limited and inconsistent reports on the relationship between $\mathrm{p} 16$ expression and response to neo adjuvant chemotherapy that consists of standard platin, taxan and 5 fluro uracil based agent in ESCC patients particularly from north east India. This study intended to find out prevalence of HPV infection as correlated by p16 expression and its impact on response to neo adjuvant chemotherapy in ESCC.

\section{Materials and Methods}

Patient Selection: This was a retrospective study involving 101 consecutive ESCC patients who reported at the Cachar Cancer Hospital Research Centre, Silchar, India from 2010-14. All of them presented with locally advanced esophageal squamous cell carcinoma and majority were in performance status 1 during initial presentation. They were therefore advised neo adjuvant chemotherapy. The chemotherapy was as per national comprehensive cancer network (NCCN) guidelines that comprised of platin, taxan and 5 fluro uracil. Institutional Review Board of Cachar Cancer Hospital \& Research Centre, Silchar, India approved (IRB/CCHRC/07/2013) this study. Formalin fixed paraffin embedded tissue blocks (FFPE) along with clinical information were used as per approved guidelines. Endoscopies and Computed Tomography were employed to stage the disease status. Endoscopic guided punch biopsies were taken for histopathology reporting and immunohistochemistry (IHC) tests.

Patients habit and medical data was extracted from the respective patient's record. Performance status of patient on first visit was assessed on Easter Cooperative Oncology Group (ECOG) scale (Oken et al., 1982).

Immunohistochemistry: Glass slide treated with 3-Aminopropyltriethoxysilane (Sigma-Aldrich) was used for adhering $4 \mu \mathrm{m}$ tissue cut section from paraffin blocks. It was left for drying under fan for 1 hour and subsequently inside incubator at $50^{\circ} \mathrm{C}$ for subsequent 12 hours or overnight. Positive controls (cervical cancer) and negative control (without primary antibody) were used during each cycle of experimentation. Slides were heated at $60^{\circ} \mathrm{C}$ for 1 hour that was followed immediately with 2 step xylene (Merck) wash, 10 minutes each for deparaffinization. Thereafter, it was rehydrated with graded alcohol for a total of 20 minutes and was kept for 1 minute in distilled water. Antigen retrieval was performed by placing slides in pre-warned $\left(65^{\circ} \mathrm{C}\right)$ Tris-EDTA (pH 9; Dako) buffer and heating it at $97^{\circ} \mathrm{C}$ for 20 minutes with 400 watt in microwave oven. After cooling it to room temperature, blockage of endogenous peroxidase was performed by immersion in 3\% hydrogen peroxide (Dako) for 10 minutes. After 5 minutes wash with phosphate buffered saline (PBS), sections were incubated with primary monoclonal antibody: p16 $6^{\mathrm{INK} 4 \mathrm{a}}$ (Clone JC8; delusion 1:150; Santa Cruz Biotechnology, USA) in humidified chamber for 1 hour. Excess primary antibody was washed with PBS. Envision Flex/HRP (Dako) was applied as secondary antibody over slides and incubated for 30 minutes in humidified chamber. After washing slides with PBS, 3,3-diaminobenzidine (Dako) was applied as chromogen for 10 minutes. Finally, sections were washed with distilled water, counterstained with haematoxyline for 45 seconds, rehydrated with graded alcohol, dried and mounted with DPX for analysis.

Immunohistochemical scoring: p16 IHC scoring was done by 2 independent pathologists blinded to this study. Strong and uniform p16 staining (both cytoplasmic and nuclear) in all or most cancer cells were considered p16 positive whereas absent or weak p16 staining in cancer cells were considered as negative (El-Naggar and Westra, 2012). Sections which shows strong p16 expression in more than $50 \%$ for tumor cells was considered as positive and less than $50 \%$ expression was considered as negative for statistical analysis.

Response assessment: Following neo adjuvant chemotherapy (NACT), patient underwent surgery. Response was assessed based on the histopathology of the resected specimen. For patients who received concurrent chemo radiotherapy following NACT, response was assessed by endoscopy and computed tomography scan. Patients who achieved complete remission were considered responder. Patients with any residue were grouped as non responders.

Statistical method: Statistical analysis was performed by using $\chi^{2}$ with likelihood ratio (LR) or Fisher's exact test for correlation analysis. Software SPSS version 10 was employed for statistical analysis. In all tests, 2 tail $P$ value was utilized. Test was considered statistically significant when $\mathrm{P} \leq 0.05$.

\section{Results}

Correlation analysis of clinicopathological factors with p16 expression in ESCC patients is shown in Tables 1 and $2.94 \%$ of patients who were reported in this study were from Barak valley in Southern Assam and were from Bengali speaking community. Over expression of p16 was reported in $22 \%$ of ESCC (22 out of 101) patients. Those 
p16 Expression as a Marker for HPV Infection in Esophageal SCC Can Predict Response to Neo-Adjuvant Chemotherapy

who had shown p16 over expression, majority (91\%) were more than 45 years old $(\mathrm{P}=0.048) .59 \%$ of $\mathrm{p} 16$ positive patients were males ( 13 out of 22$)(\mathrm{P}=0.421) .97 \%$ ESCC patients presented with dysphagia as an initial symptom. From that $22 \%$ (21 out of 97) tested positive for p16. Initial Eastern Cooperative Oncology Group (ECOG)

Table 1. Correlation analysis of clinicopathological factors with p16 expression in ESCC patients

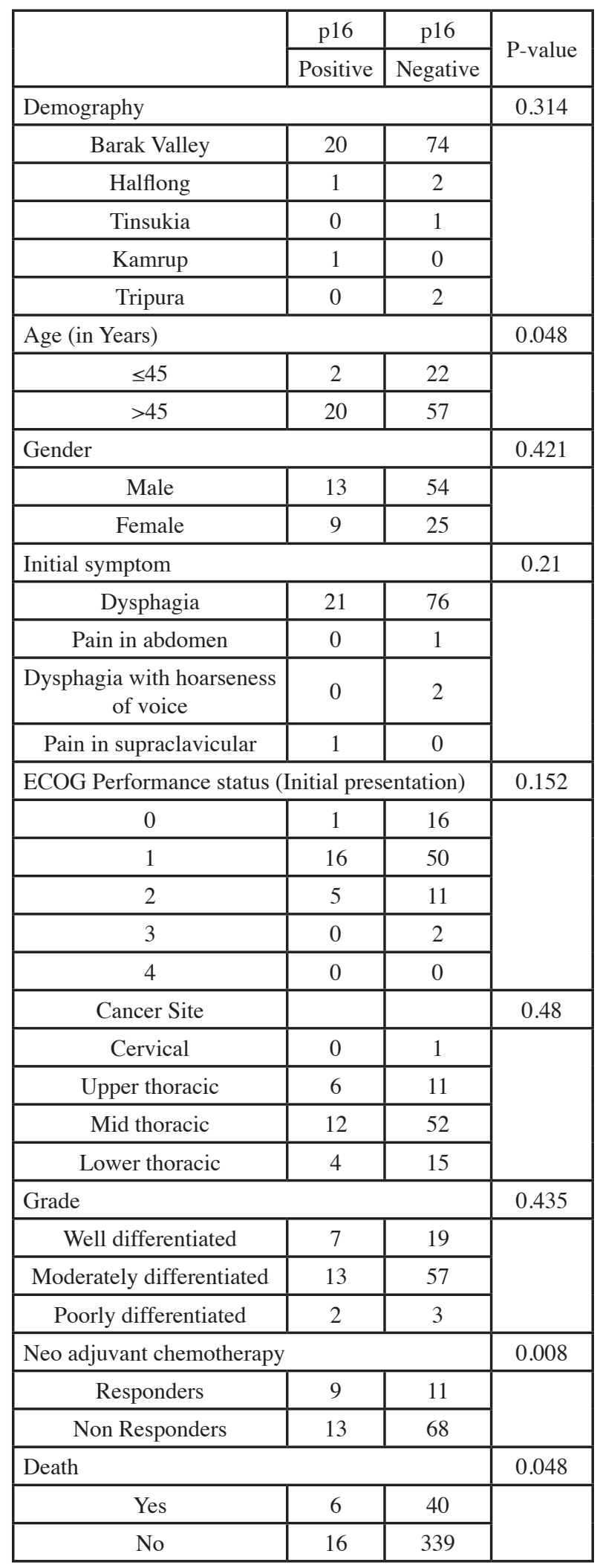

Abbreviations: ECOG = Eastern Cooperative Oncology Group
Table 2. Correlation Analysis of Reported Habits with p16 Expression in ESCC Patients

\begin{tabular}{|c|c|c|c|}
\hline & p16 Positive & p16 Negative & P-value \\
\hline \multicolumn{3}{|c|}{ Cigarette smoking } & 0.868 \\
\hline Yes & 11 & 42 & \\
\hline No & 8 & 28 & \\
\hline \multicolumn{3}{|c|}{ Tobacco chewers } & 0.313 \\
\hline Yes & 6 & 31 & \\
\hline No & 13 & 39 & \\
\hline \multicolumn{3}{|c|}{ Alcohol intake } & 0.417 \\
\hline Yes & 3 & 17 & \\
\hline No & 16 & 53 & \\
\hline \multicolumn{3}{|c|}{ Areca Nut } & 0.485 \\
\hline Yes & 15 & 60 & \\
\hline No & 4 & 10 & \\
\hline \multicolumn{3}{|l|}{ Pan } & 0.297 \\
\hline Yes & 13 & 56 & \\
\hline No & 6 & 14 & \\
\hline
\end{tabular}

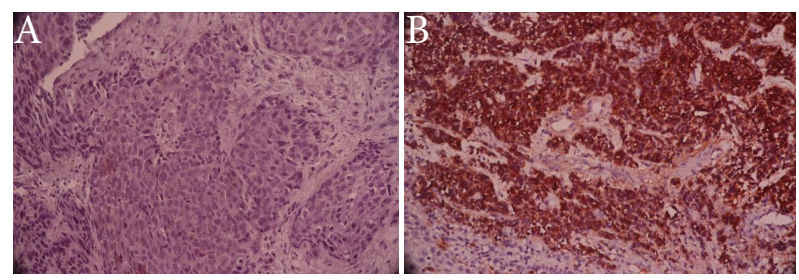

Figure 1.p16 Expression in ESCC.A) p16 negative ESCC tumor; B) p16 positive ESCC tumor

performance status was reported either 0 (normal) or 1 (near normal) or 2 (difficulty in normal activity) in $17 \%$, $66 \%$ and $16 \%$ patients respectively. p16 positive tumors appeared more prominently in upper two third of thoracic region (82\%) and tended to expressed more in moderately differentiated ESCC (59\%). However, ESCC tumors that were found positive for $\mathrm{p} 16$ expression appeared to fall into responders group rather than non responders $(\mathrm{P}=0.008)$ and reported with less mortality $(\mathrm{P}=0.048)$. $41 \%$ p16 positive were found responders whereas $45 \%$ responders were observed $\mathrm{p} 16$ positive.

p16 positive staining was observed in 22 cases as represented in Figure 1. Information related to habit was available for only $89 \mathrm{ESCC}$ patients. Out of which $21 \%$ were positive for p16 and remaining $79 \%$ were negative. Predominantly $84 \%$ were reported using areca nut, $60 \%$ smokers, $42 \%$ tobacco chewers, $77 \%$ pan users and merely $22 \%$ remarked alcohol intake.

\section{Discussion}

Our understanding of the role of HPV in carcinogenesis is evolving. It is strongly implicated as a causative factor in the tumorogenesis of squamous cell carcinoma of cervix (Walboomers et al., 1999; Hausen, 2009). There is a strong association of HPV with squamous cell cancer of the head and neck, commonly oropharynx (D'Souza et al., 2007), where p16 expression act as predictive marker for 
response to radiation therapy. Its role in ESCC (Liyanage et al., 2013) is unclear. However, it is implicated in some studies as predictive and prognostic marker of squamous cell carcinomas (Lassen et al., 2009; Fischer et al., 2010; Cao et al., 2014).

Prevalence of HPV as evidenced by p16 over expression in ESCC was found in 22\% (22 out of 101) of studied cases in our study. It correlates with the results of a recent meta analysis of 13832 ESCC patients involving 124 studies, where the average prevalence of HPV was found $0.304(0.185,0.423)$ by immunohistochemistry with $95 \%$ confidence interval (Petrick et al., 2014). A similar meta analysis based on 132 studies related to HPV in ESCC by Hardefeldt HA found HPV prevalence in ESCC at $24.8 \%$ (Hardefeldt et al., 2014).

However there exists inconclusive evidence of its aetiological significance in the development of ESCC (Mohiuddin et al., 2013; Zhang et al., 2014). Few Meta studies though have reported that HPV infection can increase the risk of developing ESCC by 3 folds (Liyanage et al., 2013).

In this study male ESCC patients were reported approximately two times more than their female counterpart with a male to female ratio of $1.97: 1$. This is similar to literature report of male to female ratio of 1.8:1 (Mohiuddin et al., 2013). p16 expression was however more common in ESCC in women's. We reported $26 \%$ of ESCC in females and $19 \%$ in males overexpressed p16 $(\mathrm{P}=0.421)$ in this study.

This study reported $69 \%$ ESCC tumors were in moderately differentiated grade where $19 \%$ were shown p16 positivity. In squamous cell carcinoma of cervix, p16 positivity has been reported in $100 \%$ of patients (Ma et al., 2010). We found HPV infection was more prevalent in upper thoracic region (35\%) than mid (19\%) and lower thoracic region $(21 \%)$ that may be due to its proximity to oropharyngeal squamous epithelium, which acts as route for HPV infection. Similar result was also reported by Fangli et al., 2014.

We found $43 \%$ those with pathologically complete response were 16 positive whereas $16 \%$ non responders positive for $\mathrm{p} 16$. In this study we have found $\mathrm{p} 16$ expression more common in responders $(\mathrm{P}=0.009)$, which indicates that p16 correlated HPV positive tumors show favourable outcome in terms of reaching pathologically complete response (PCR) of tumor after the complete regime of neo adjuvant chemotherapy. Patients who shown p16 positivity were reported less deaths (27\%) and among all reported deaths, majority $87 \%$ were p16 negative $(\mathrm{P}=0.048)$. This correlates with similar finding where HPV positivity was shown to increase progression free survival and disease free survival in patients with squamous cell carcinomas (Ang et al., 2010; Fischer et al., 2010; Fangli et al., 2014).

The role of tobacco and areca nut chewing, tobacco smoking and alcohol drinking have shown to be a risk factor in the tumorogenesis of different types of squamous cell carcinoma including ESCC (Lee et al., 2005; Ko et al., 1995; Muwonge et al., 2008; Lagergren and Lagergren 2010; Pramesh et al., 2015). Although the prevalence of areca nut $(84 \%)$, pan $(77 \%)$, cigarette $(60 \%)$, tobacco using (42\%), were high in ESCC patients, we found no statistical significance of these factors with p16 expression. Extensive use of areca nut and tobacco in this region corresponds to the finding of highest esophageal cancer AAR of 71.4 in males and 30.2 in females reported in East Khasi Hills of Meghalaya state in north east India whereas in Asia it reported second highest in China, Jiashan at AAR 20.2 for males and in Pakistan, South Karachi at AAR 8.6 for females (NCRP 2013).

The percentage of alcohol use among ESCC patients was found considerable low at $22 \%$ in our study. It show no correlation with the available report where alcohol was considered as one of the important risk factor for developing ESCC (Blot, 1999; Lagergren and Lagergren 2010).

Current data though suggests the role of p16 as predictive marker for treatment response assessment however a similar study on a larger number of ESCC patients will provide further evidence that is required to implement this finding in routine clinical settings for better management of ESCC.

\section{Acknowledgements}

DBT (Gov. of India) for funding.

\section{References}

Ali I, Wani WA, Saleem, K (2011). Cancer scenario in India with future perspectives. Cancer Ther, 8, 56-70.

Ang KK, Harris J, Wheeler R, et al (2010). Human papillomavirus and survival of patients with oropharyngeal cancer. $N$ England J Med, 363, 24-35.

Cao F, Zhang W, Zhang F, et al (2014). Prognostic significance of high-risk human papillomavirus and $\mathrm{p} 16^{\mathrm{INK} 4 \mathrm{~A}}$ in patients with esophageal squamous cell carcinoma. Int J Clin Exp Med, 7, 3430-8.

Cao F, Han H, Zhang F, et al (2014). HPV infection in esophageal squamous cell carcinoma and its relationship to the prognosis of patients in northern China. Scientific World J, 2014, 1-9.

Chung CS, Lee YC, Wang CP, et al (2010). Secondary prevention of esophageal squamous cell carcinoma in areas where smoking, alcohol, and betel quid chewing are prevalent. $J$ Formos Med Assoc, 109, 408-21.

de Villiers EM, Lavergne D, Chang F, et al (1999). An interlaboratory study to determine the presence of human papillomavirus DNA in esophageal carcinoma from China. Int J Cancer, 81, 225-8.

El-Naggar AK, Westra WH (2012).p16 expression as a surrogate marker for HPV-related oropharyngeal carcinoma: a guide for interpretative relevance and consistency. Head Neck, 34, 459-61.

Fischer CA, Kampmann M,Zlobec I, et al (2010). p16 expression in oropharyngeal cancer: its impact on staging and prognosis compared with the conventional clinical staging parameters. Ann Oncol, 21, 1961-6.

Hardefeldt HA, Cox MR, Eslick GD (2014). Association between human papillomavirus (HPV) and oesophageal squamous cell carcinoma: a meta-analysis. Epidemiol Infection, 142, 1119-37.

International Agency for Research on Cancer (2014). Available: http://globocan.iarc.fr/Pages/fact_sheets_cancer.aspx.

Kalof AN, Cooper K (2006). p16INK4a immunoexpression: surrogate marker of high-risk HPV and high-grade cervical intraepithelial neoplasia. Adv Anat Pathol, 13, 190-4. 
Katiyar S, Hedau S, Jain N, Kar, et al (2005). p53 gene mutation and human papillomavirus (HPV) infection in esophageal carcinoma from three different endemic geographic regions of India. Cancer Lett, 218, 69-79.

Lagergren J, Lagergren P (2010). Oesophageal cancer. BMJ, 21, 341-6280.

Liyanage SS, Rahman B, Ridda I, et al (2013). The aetiological role of human papillomavirus in oesophageal squamous cell carcinoma: a meta-analysis. Plos One, 8, 1-12.

Ma YY, Ye F, Chen XD, et al (2010). Evaluation of P16(INK4 $\alpha)$ expression in Thinprep cervical specimens for the predication of high-grade cervical intraepithelial neoplasia. Zhonghua Yi Xue Za Zhi, 90, 3040-4.

Mohiuddin MK, Chava S, Upendrum P, et al (2013). Role of human papilloma virus infection and altered methylation of specific genes in esophageal cancer. Asian Pac J Cancer Prev, 14, 4187-93.

Murthy NS, Chaudhry K, Rath GK (2008). Burden of cancer and projections for 2016, Indian scenario: gaps in the availability of radiotherapy treatment facilities. Asian Pac J Cancer Prev, 9, 671-77.

Murthy NS, Nandakumar BS, Shivaraj NS, et al (2010). Cancer registration: its relevance for health care planning in india. Indian J Prev Soc Med, 41, 75-87.

National Cancer Registry Program (2013). Available at http:// www.ncrpindia.org/Annual_Reports.aspx

Petrick JL, Wyss AB, Butler AM, et al (2014). Prevalence of human papillomavirus among oesophageal squamous cell carcinoma cases: systematic review and meta-analysis. $B R$ J Cancer, 110, 2369-77.

Pramesh CS, Karimundackal G, Jiwnani S (2015). Squamous cell carcinoma of the oesophagus. in 'esophageal squamous cell carcinoma', Springer Japan, 279-303.

Sanne Hoxbroe Michaelsen, Christian Gronhoj Larsen, Christian von Buchwald (2014). Human papillomavirus shows highly variable prevalence in esophageal squamous cell carcinoma and no significant correlation to p16ink4a overexpression a systematic review. J Thorac Oncol, 9, 865-71.

Shukla S, Bharti AC, Mahata S, et al (2009). Infection of human papillomaviruses in cancers of different human organ sites. Indian J Med Res, 130, 222-33.

Souza GD, Kreimer AR, Viscidi R, et al (2007). Case-control study of human papillomavirus and oropharyngeal cancer. $N$ England J Med, 356, 1944-56.

Syrjanen K, Pyrhonen S, Aukee S, Koskela E (1982). Squamous cell papilloma of the esophagus: a tumour probably caused by human papilloma virus (HPV). Diagn Histopathol, 5, 291-6.

Syrjanen KJ (2002). HPV infections and oesophageal cancer. $J$ Clin Pathol, 55, 721-8.

Takiar R, Nadayil D, Nandakumar A (2010). Projections of number of cancer cases in India (2010-2020) by cancer groups. Asian Pac J Cancer Prev, 11, 1045-49.

Vaiphei K, Kochhar R, Bhardawaj S, et al (2013). High prevalence of human papillomavirus in esophageal squamous cell carcinoma: a study in paired samples. Dis Esophagus, 26, 282-7.

Walboomers JM, Jacobs MV, Manos MM, et al (1999). Human papillomavirus is a necessary cause of invasive cervical cancer worldwide. J Pathol, 189, 12-9.

Zhang SK, Guo LW, Chen Q, et al (2014). Prevalence of human papillomavirus 16 in esophageal cancer among the Chinese population: a systematic review and meta-analysis. Asian Pac J Cancer Prev, 15, 10143-49.

Zur Hausen H (2009). Papillomaviruses in the causation of human cancers - a brief historical account. Virol, 384, 260-5. 